\section{Questions} vives

\section{Questions Vives}

Recherches en éducation

Vol. $5 n^{\circ} 11 \mid 2009$

Le développement professionnel : quels indicateurs ?

\title{
Piot, T., Marcel J.-F. \& Tardif, M. (Eds.), Le travail partagé des enseignants
}

Les Sciences de l'Éducation Pour l'Ère Nouvelle, 42 (2), 2009

\section{Julie Blanc}

\section{OpenEdition}

\section{Journals}

Édition électronique

URL : http://journals.openedition.org/questionsvives/635

DOI : $10.4000 /$ questionsvives. 635

ISBN : 978-2-8218-1082-2

ISSN : 1775-433X

Éditeur

Université Aix-Marseille (AMU)

Édition imprimée

Date de publication : 1 janvier 2009

Pagination : $316-318$

ISBN : 978-2-912643-35-3

ISSN : 1635-4079

Référence électronique

Julie Blanc, «Piot, T., Marcel J.-F. \& Tardif, M. (Eds.), Le travail partagé des enseignants », Questions Vives [En ligne], Vol. $5 n^{\circ} 11$ | 2009, mis en ligne le 01 juin 2011, consulté le 22 septembre 2020. URL http://journals.openedition.org/questionsvives/635; DOI : https://doi.org/10.4000/questionsvives.635

Ce document a été généré automatiquement le 22 septembre 2020.

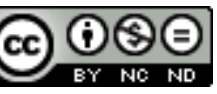

Questions Vives est mis à disposition selon les termes de la licence Creative Commons Attribution -

Pas d'Utilisation Commerciale - Pas de Modification 4.0 International. 


\section{Piot, T., Marcel J.-F. \& Tardif, M. (Eds.), Le travail partagé des enseignants}

Les Sciences de l'Éducation Pour l'Ère Nouvelle, 42 (2), 2009

Julie Blanc

\section{RÉFÉRENCE}

Piot, T., Marcel J.-F. \& Tardif, M. (Eds.) (2009). Le travail partagé des enseignants. Les Sciences de l'Éducation Pour l'Ère Nouvelle, 42(2).

1 Ce numéro thématique sur le travail partagé vient ponctuer une série d'ouvrages témoignant de la nécessité de mettre au jour une face souvent méconnue et peu explorée dans le champ de la recherche en éducation, à savoir le travail partagé des enseignants. Il pose à nouveau que les pratiques professionnelles de l'enseignant ne sont plus circonscrites à la relation pédagogique avec les élèves en classe, à l'image de l'enseignant "solitaire ", mais comprennent également les activités réalisées avec d'autres acteurs (enseignants, partenaires extérieurs, acteurs de l'établissement...). Le travail partagé étant fortement lié au contexte socio-historique de l'établissement, il n'est pas forcément dépendant des prescriptions institutionnelles et repose sur l'engagement individuel des enseignants concernés. L'introduction du numéro est d'ailleurs consacrée à une définition relativement complète du travail partagé qui constitue l'épine dorsale de l'ensemble des contributions présentées. Cette définition souligne en outre, les liens d'interdépendance des pratiques enseignantes de travail partagé et des pratiques d'enseignement.

2 René Amigues, après avoir rappelé la pertinence de la mobilisation des sciences du travail pour étudier l'activité enseignante, pose que travailler est une activité forcément partagée dans le temps et dans l'espace, d'une part ; avec les autres, d'autre part. Il investigue le champ de la prescription éducative qu'il considère comme un 
artefact culturel dans la mesure où elle est consubstantielle de l'activité. Ses travaux indiquent que la prescription, loin de constituer un "prêt à agir " pour l'enseignant, provoque une activité de re-conception, de transformation médiée par un «questionnement professionnel » au sein du collectif de travail. Pour lui, l'écart entre le prescrit et le réalisé, mis au jour par la clinique de l'activité, incarne l'espace dans lequel les enseignants construisent de façon collective des outils, des dispositifs nouveaux; en somme, conçoivent les moyens à mettre en œuvre pour réaliser la prescription. Celle-ci constitue donc un organisateur du travail partagé des enseignants qui s'actualiserait variablement selon les établissements et qui entraînerait de facto une source de développement et d'apprentissage dépendante des milieux de travail.

3 S'appuyant sur une enquête empirique menée avec des enseignants de CE2 autour du dispositif des évaluations nationales, J.-F. Marcel et A. Garcia montrent l'existence d'interrelations entre les pratiques d'enseignement et les activités professionnelles collaboratives. La recherche révèle des différences significatives dans les pratiques d'enseignement en fonction des modalités d'organisation du travail partagé mises en œuvre par les collectifs d'enseignants dans les écoles. Un lien est donc dégagé entre la dynamique à l'œuvre dans l'école dans la mise en place d'activités collaboratives et la dynamique pédagogique que les enseignants insufflent dans leur classe avec leurs élèves. Les théories de l'apprentissage social (Bandura, 1976; Perret-Clermont, 1996; Doise et Mugny, 1997) interviennent alors comme un cadre explicatif de ces interrelations ; particulièrement l'apprentissage par imitation active et l'apprentissage par conflit sociocognitif. Les éléments empiriques qui ont servi d'illustration au cadre explicatif ont aussi permis d'affiner la définition du travail partagé en élargissant la notion de partage à différentes formes de médiation telles que l'emprunt de supports pédagogiques.

4 L'acception de travail partagé est cependant relativement large, en témoignent les travaux de $\mathbf{C}$. Mérini et $\mathbf{P}$. Ponte dont la problématique porte davantage sur le travail conjoint. Si le travail partagé relève des échanges et des activités collaboratives hors de l'espace de la classe dans les travaux cités précédemment; ici, il s'agit de pratiques d'enseignement conjointement menées en classe par l'enseignant et un maître surnuméraire. À partir d'une enquête sociologique, l'objectif a été d'identifier les règles d'action communes, leur émergence, les stratégies développées par les acteurs et ainsi d'appréhender les continuités et discontinuités de sens dans les actions. Les chercheures mettent en évidence que le travail conjoint aboutit à une mise en réseau des pratiques, lesquelles induisent des compétences professionnelles spécifiques.

Dans l'article suivant, T. Piot évoque un autre exemple de travail partagé en présence d'élèves: la co-intervention de deux enseignants face à une même classe dans le contexte des itinéraires de découvertes développés au collège. Mobilisant la notion d'image opérative (Ochanine, 1978), sa recherche permet d'inférer des processus d'ajustement en tension pour coordonner le travail conjoint des enseignants. L'un résulte d'une coordination anticipatrice au travers de la production d'une image opérative de référence partagée, celle-ci servirait de base d'orientation commune à l'activité conjointe des deux enseignants. L'autre processus relève d'une coordination (régulation) qui se déroule en cours d'action, conséquence d'ajustements interactifs et réciproques de l'image effectrice partagée ; celle-ci viserait à rendre la co-intervention fluide, cohérente et synchronisée facilitant le travail et la compréhension des élèves des objectifs visés. 
$6 \quad \mathbf{M}$. Tardif et $\mathbf{C}$. Borges concluent ce recueil de travaux en proposant une synthèse des différentes facettes du travail partagé. Ils inscrivent les activités collaboratives au centre d'une nouvelle professionnalisation et d'un processus mouvant, occupant de plus en plus de place dans les pratiques enseignantes, du fait de multiples facteurs: réformes, évolution des établissements et des publics, essor de nouveaux personnels scolaires et transformations du métier enseignant (des missions, des savoirs...). L'intérêt de l'article repose sur une nouvelle mise en perspective, à savoir l'ambiguïté du terme «partage " qui signifie non seulement "mise en commun» mais aussi "division ", "séparation ». Leurs travaux mettent d'ailleurs en exergue une tendance historique à la division du travail induite notamment par la multiplication des agents scolaires. Ceci les conduit donc à étudier, outre les conditions du «faire ensemble », les résistances objectives au travail partagé.

\section{AUTEURS}

\section{JULIE BLANC}

Toulouse EducAgro - ENFA, Université de Toulouse 